\title{
Risk of haemolytic uraemic syndrome caused by shiga-toxin- producing Escherichia coli infection in adult women in Japan
}

\author{
J. FUJII ${ }^{*}$, T. MIZOUE ${ }^{2}$, T. KITA ${ }^{3}, \mathrm{H} . \mathrm{KISHIMOTO}^{4}, \mathrm{~K} \cdot \mathrm{JOH}^{4}$, Y. NAKADA ${ }^{5}$,

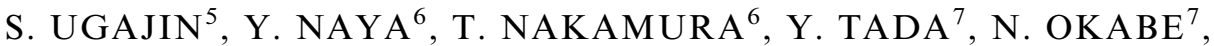 \\ Y. MARUYAMA ${ }^{8}, \mathrm{~K}$. SAITOH ${ }^{9}$ AND Y. KUROZAWA ${ }^{10}$ \\ ${ }^{1}$ Division of Bacteriology, Department of Microbiology and Immunology, Faculty of Medicine, Tottori \\ University, Yonago, Japan; ${ }^{2}$ Department of Epidemiology and International Health, Research Institute, \\ International Medical Center of Japan, Toyama, Shinjuku-ku, Tokyo, Japan; ${ }^{3}$ Department of Forensic Medicine, \\ School of Medicine, University of Occupational and Environmental Health, Kitakyushu, Japan; ${ }^{4}$ Saitama \\ Children's Medical Center, Magome, Iwatsuki, Saitama, Japan; ${ }^{5}$ Utsunomiya City Institute of Public Health and \\ Environment, Takebayashimachi, Utsunomiya, Tochigi, Japan; ${ }^{6}$ Utsunomiya City Public Health Office, \\ Takebayashimachi, Utsunomiya, Tochigi, Japan; ${ }^{7}$ Infectious Disease Surveillance Center (IDSC), National \\ Institute of Infectious Diseases (NIID), Toyama, Shinjuku-ku, Tokyo, Japan; ${ }^{8}$ Kagawa Prefectural Chusan \\ Regional Health and Welfare Office, Dokichohigashi, Marugame, Kagawa, Japan; ${ }^{9}$ Kagawa Prefectural Shozu \\ Regional Health and Welfare Office, Fuchisakikou, Tonoshocho, Shozugun, Kagawa, Japan; ${ }^{10}$ Division of Health \\ Administration and Promotion, Department of Social Medicine, Faculty of Medicine, Tottori University, Yonago, Japan
}

Received 13 January 2015; Final revision 12 August 2015; Accepted 8 September 2015; first published online 16 October 2015

\section{SUMMARY}

Shiga-toxin-producing Escherichia coli (STEC) infections usually cause haemolytic uraemic syndrome (HUS) equally in male and female children. This study investigated the localization of globotriaosylceramide $(\mathrm{Gb} 3)$ in human brain and kidney tissues removed from forensic autopsy cases in Japan. A fatal case was used as a positive control in an outbreak of diarrhoeal disease caused by STEC O157:H7 in a kindergarten in Urawa in 1990. Positive immunodetection of Gb3 was significantly more frequent in female than in male distal and collecting renal tubules. To correlate this finding with a clinical outcome, a retrospective analysis of the predictors of renal failure in the 162 patients of two outbreaks in Japan was performed: one in Tochigi in 2002 and the other in Kagawa Prefecture in 2005. This study concludes renal failure, including HUS, was significantly associated with female sex, and the odds ratio was 4.06 compared to male patients in the two outbreaks. From 2006 to 2009 in Japan, the risk factor of HUS associated with STEC infection was analysed. The number of males and females and the proportion of females who developed HUS were calculated by age and year from 2006 to 2009. In 2006, 2007 and 2009 in adults aged $>20$ years, adult women were significantly more at risk of developing HUS in Japan.

Key words: Gb3, haemolytic uraemic syndrome, HUS, STEC.

\section{INTRODUCTION}

In May 2011, a large outbreak of Shiga toxin (Stx)producing Escherichia coli (STEC) O104:H4 occurred

\footnotetext{
* Author for correspondence: Dr J. Fujii, Division of Bacteriology, Department of Microbiology and Immunology, Faculty of Medicine, Tottori University, 86 Nishi-cho, Yonago City, Japan (Email: junfujii@med.tottori-u.ac.jp)
}

in Germany, resulting in more than 4000 patients in 16 countries across Europe and North America $[1,2]$. This outbreak caused 855 cases of haemolytic uraemic syndrome (HUS) and 54 deaths [3]. A total of $68 \%$ of the patients with HUS were adult women [4]. However, Werber et al. reported that adult women had higher odds of developing HUS [odds ratio (OR) 1.83] in the outbreak of STEC O104:H4 
[5], but this was not statistically significant. HUS consists of the triad of acute haemolytic anaemia, thrombocytopenia, and acute renal dysfunction [6]. In Japan, the first outbreak of STEC O157:H7 infection occurred in 174 children in a nursery school in Saitama. The bacterium was isolated from tap water supplied from a well at the school. Fourteen cases developed complete HUS defined by uraemia, anaemia and thrombocytopenia, while six cases developed incomplete HUS and two boys died with intractable generalized seizures within 3 days after onset of diarrhoea [7]. Between May and December 1996, there were 9451 cases and 12 deaths from STEC O157:H7, representing 16 total outbreaks involving $>10$ patients each, including the Sakai City incident [8] in July 1996, where an outbreak of STEC O157:H7 infection occurred in schoolchildren. Lunch foods supplied in the elementary schools in Sakai were found to be contaminated with STEC O157:H7. One hundred and twenty-one cases of HUS developed in 12680 symptomatic patients, including putative secondary infections, and three girls died during the outbreak [9]. In this large outbreak, sex was not associated with progression to complete or incomplete HUS [10]. Moreover, in 1996, an outbreak in Central Scotland involved the hospitalization of 120 patients [11]. In that outbreak 34 patients developed HUS; 28 of whom were adults, and 16 deaths in adults were associated with STEC O157:H7 infection. Progression to HUS was also not associated with sex in that outbreak [11]. In 2002, two outbreaks of STEC O157:H7 infection occurred in patients in a psychiatric inpatient unit and residents of care homes for the elderly in Utsunomiya, Tochigi Prefecture, Japan. One hundred and twenty-three individuals developed diarrhoea or bloody diarrhoea, five developed HUS, and nine died. In 2005, two clusters of diarrhoea occurred in patients in two care homes for the elderly in Kagawa Prefecture, Japan. Of 263 elderly residents, 39 developed a gastrointestinal illness including bloody diarrhoea, five developed HUS, and seven patients died. STEC O157 was isolated from the patients with diarrhoea and the pickled Chinese cabbage provided at breakfast and dinner. From clinical signs and laboratory data, many useful predictive clinical mortality risk factors were investigated during this outbreak.

The Stx family, including Stx1 and Stx2, comprises $1 \mathrm{~A}$ and $5 \mathrm{~B}$ subunit proteins. The A subunit has $\mathrm{N}$-glycosidase activity that removes adenine 4324 of
28S RNA of the 60S ribosomal subunit [12], rendering ribosomes inactive for protein synthesis. Each B subunit binds with high affinity to the glycosphingolipid globotriaosylceramide (Gb3), which is present in specific host/mammalian cells [13]. Gb3 is identical to CD77 antigen as a marker for B-cell Burkitt's lymphoma [14]. This study focused on detecting the localization of the Stx receptor, Gb3, in human brain and kidney tissues removed from 33 autopsy cases in Japan. The relationship between the presence of Gb3 and the sex and age of humans was analysed. To confirm the results of this experiment, a retrospective analysis of predictors of renal failure in the combined infected patients who suffered from a gastrointestinal illness including diarrhoea or bloody diarrhoea admitted to the hospital during the two above outbreaks in Kagawa and Tochigi prefectures was performed.

Finally, the Infectious Disease Surveillance Center (IDSC) of the National Institute of Infectious Diseases (NIID) in Japan has recorded the number of cases of HUS since 1996. This study analysed the sex and age distribution of HUS from 2006 to 2009 in Japan.

\section{MATERIALS AND METHODS}

\section{Localization of CD77 in human brain and kidney from forensic autopsies}

Human brain and kidney tissues were obtained from forensic autopsies of 33 individuals, including 20 adults (10 male, 10 female) and 13 infants ( 7 male, 6 female), all without brain tumours, brain lesions or renal dysfunction. The average age of adults and infants was $46 \cdot 6 \pm 12 \cdot 3$ years and $10 \cdot 1 \pm 8.68$ months, respectively. The causes of death were loss of blood $(50 \%)$ or haemorrhagic shock $(50 \%)$ in adults, and sudden infant death syndrome $(23 \%)$, asphyxia $(38.5 \%)$ or unknown $(38.5 \%)$ in infants. In all cases, the interval between death and autopsy was $<24 \mathrm{~h}$. Tissues were sectioned ( $5 \mu \mathrm{m}$ thick), fixed for 3 days in $10 \%$ formalin, and embedded in paraffin. To detect Gb3 immunohistochemically, monoclonal rat antihuman CD77 antibody (Immunotech, USA) was used. The peroxidase-conjugated streptavidin-biotin method was used for staining of paraffin-embedded sections. Using the immunohistochemical sections from each autopsy, Gb3-positive tissues were discovered. The percentage of autopsy cases which indicated a positive immunodetection of $\mathrm{Gb} 3$ was calculated 
with an adult denominator of 10 males and 10 females and an infant denominator of 7 males and 6 females. Comparisons of the Gb3-positive cases (adult vs. infant, male vs. female) were performed by Fisher's exact two-tailed test. The receptor for Stx was detected in paraffin-embedded sections. It became apparent that the glycolipid antigen Gb3 was detectable and remained in spite of exposure of tissue to processing reagents such as alcohol and xylene utilized in the deparaffinization procedure [15]. As a positive control, paraffin-embedded kidney and brain sections were obtained at autopsy from a 4-year-old boy with incomplete HUS and central nervous manifestation who subsequently died from STEC infection in Saitama Children's Medical Centre in 1990 [7]. All the procedures were performed according to the guidelines of the Japanese Society of Legal Medicine.

\section{Retrospective analysis of predictors of death in infected patients admitted to the hospital in two care homes for the elderly in Kagawa Prefecture, Japan}

For all patients admitted to hospital, data such as premorbid illness, antibiotic therapy, symptoms, signs, management, and complications were collected from case records. The study included all patients with confirmed and probable cases of STEC infection who were admitted to the hospital during the outbreak. Patients were considered confirmed cases if the outbreak strain was isolated from their stool sample using the standard culture method. Patients were considered to be probable cases if they had bloody diarrhoea or HUS and had eaten the implicated pickled Chinese cabbage. Complete HUS was defined as follows: evidence of anaemia (haemoglobin level $<10 \mathrm{~g} / \mathrm{dl}$ ), thrombocytopenia (platelet count $<10^{6}$ cells/l), and acute renal impairment (serum creatinine level greater than reference value for age). Incomplete HUS was defined by any two of the criteria for complete HUS. Demographic characteristics including age and sex, presence or absence of bloody diarrhoea, detection of STEC in stool specimens, bedridden status, laboratory variables, and antibiotic therapy were assessed in relation to outcome measures of death by statistical analysis. Statistical significance for the difference in frequency between categorical variables was assessed by Fisher's two-tailed exact test. For bedridden status, logistic regression analysis was done to estimate the OR and its $95 \%$ confidence interval (CI) for the association between exposure and outcome. Laboratory data was monitored for all possible patients who were admitted to hospital. White blood cell (WBC) count, haemoglobin, urea, and creatinine were assessed as predictors of death based on the results from the first blood sample obtained. Judgement on the degree of bedridden status in disabled elderly persons was determined [16]. J1 and J2 signify that the patients are independent - capable of leaving the house and have a high degree of mobility; A1 and A2 signify the patients are partially bedridden - generally housebound; B1 and B2 signify the patients are completely bedridden; and $\mathrm{C} 1$ and $\mathrm{C} 2$ signify the patients are completely bedridden and impossible to turn over in bed.

Antibiotic therapy for gastroenteritis was prescribed according to Japanese guidelines. Fosfomycin or levofloxacin was given to 16 patients in care home no. 1 . The study was approved by the Ethics Committee of Kyushu University Faculty of Medical Sciences.

\section{Retrospective statistical analysis of risk factors for HUS or renal failure caused by STEC in Japan}

Consistent with the results of the immunodetection of Gb3 in human autopsy cases, the study performed a retrospective analysis of the predictors of renal failure in the combined 162 infected patients during the two outbreaks. In the 2002 outbreak in Tochigi Prefecture, 123 patients developed diarrhoea or bloody diarrhoea, including five cases of HUS and nine patients who died. In another outbreak of STEC infection in Kagawa Prefecture in 2005, 39 patients developed diarrhoea or bloody diarrhoea, including five who developed HUS and seven who died. In the present study, 162 cases had gastrointestinal symptoms, including diarrhoea or bloody diarrhoea. Eight patients had complete HUS, 17 had renal failure, including complete or incomplete HUS, and 17 who were admitted to hospital died. Retrospective statistical analysis was performed. The difference in outcome measures (renal failure including HUS and death) between female and male patients was tested statistically using the Mantel-Haenszel method to adjust for age group $(<60,60-69,70-79, \geqslant 80$ years). Outcome measures included HUS or renal failure (serum creatinine level greater than reference value for age). Complete HUS was defined as above. The median age of the study patients was $68 \cdot 8 \pm 19 \cdot 1$ years, and $59 \%$ were female. The study was approved 
by the Ethics Committee of Kyushu University Faculty of Medical Sciences.

\section{Sex distribution of HUS patients infected with STEC from 2006 to 2009 in Japan}

Due to the small number of adult HUS cases in the study, it was determined necessary to carry out further confirmation, based on surveillance data published by the IDSC in Japan, in order to demonstrate the link between female sex and STEC infection. The IDSC have performed surveillance of HUS in Japan every year since 1996 . The study analysed sex and age distribution of HUS in Japan from 2006 to 2009. The number of males and females and the proportion of females who developed HUS were calculated by age $(<5,5$ to $<20$ years, $\geqslant 20$ years) and calendar year (2006-2009). The stool samples of all HUS cases were tested for the presence of STEC infection. One-sample test of proportion was used to test the difference of sex for each combination of age group and calendar year, as well as for their subtotal.

\section{Statistical analysis}

All analysis was performed using Stata version 10.1 (StataCorp., USA).

\section{RESULTS}

\section{Localization of CD77 in human brain and kidney from forensic autopsies}

This study analysed the relationship between the presence of Gb3 and sex and age. Gb3 immunoreactivity was localized in the Purkinje cells in the cerebellum, blood vessels, neurons and the ependymal cell layers surrounding the ventricle (Fig. 1). Positive immunodetection of Gb3 in blood vessels and neurons was more frequent in infant brain tissues than in adult brain tissues (Table 1). In the kidney samples, Gb3 was more readily detectable in mesangial cells of infants than adults $(P<0 \cdot 01)$ (Table 1$)$. Females had a more frequent positive reaction than males for Gb3 in the distal tubules and collecting tubules of the kidney (Table 1). In the positive controls, Gb3 was detected in Purkinje cells, neurons, brain microvascular endothelial cells, and mesangial cells of the kidney sample in the fatal case of a 4-year-old boy in Urawa in 1990 (Fig. 1).
Retrospective analysis of predictors of death in infected patients admitted to hospital in two care homes for the elderly in Kagawa Prefecture, Japan

In this study, 52 people with confirmed and probable cases of STEC infection were identified by stool culture. Thirty-nine patients had gastrointestinal symptoms including diarrhoea or bloody diarrhoea. Thirteen residents were asymptomatic with positive stool culture. Date of onset of illness ranged from 12 October to 2 November 2005. Three patients had complete HUS, two had an incomplete form of HUS, and all five patients with HUS who died had neurological complications. The mortality rate in symptomatic patients was $18 \%(7 / 39)$. Seven of the hospitalized patients died. Bloody diarrhoea was significantly associated with death (Table 2). When logistic regression analysis was carried out, with adjustment for age, sex and home, completely bedridden status ( $\mathrm{C} 1$ and $\mathrm{C} 2)$ was significantly associated with death (OR $8 \cdot 4,95 \%$ CI $1 \cdot 2-57 \cdot 2, P=0 \cdot 023$ ). By univariate analysis, WBC count $>1.02 \times 10^{10}$ cells $/ 1$ $(P=0.039)$ was significantly associated with death (Table 3). In care home no. 1, 19 (73\%) of 26 of the hospitalized patients were treated with levofloxacin or fosfomycin after onset of symptoms, and seven $(27 \%)$ of 26 patients had no antibiotic treatment. This difference did not reach significance (Table 3).

\section{Retrospective statistical analysis of risk factors for HUS or renal failure caused by STEC in Japan}

The association between sex and renal failure in two outbreaks is shown in Table 4. Renal failure occurred in 15/95 female patients and in 2/67 men. Logistic regression analysis with adjustment for age was done to investigate the relationship between sex and renal failure or death due to STEC infection.

Renal failure including HUS was significantly associated with female sex (OR 4.06 with adjustment for age, $P=0.046$ ). Death occurred in $14 / 95$ female patients while in male patients the number of deaths was $2 / 67$; however, sex was not significantly associated with death (OR 3.61 with adjustment for age $P=0 \cdot 11)$.

\section{Sex distribution of HUS patients infected with STEC from 2006 to 2009 in Japan}

For adults aged $\geq 20$ years in 2006, 2007, and 2009, HUS was significantly associated with female sex 
(a)
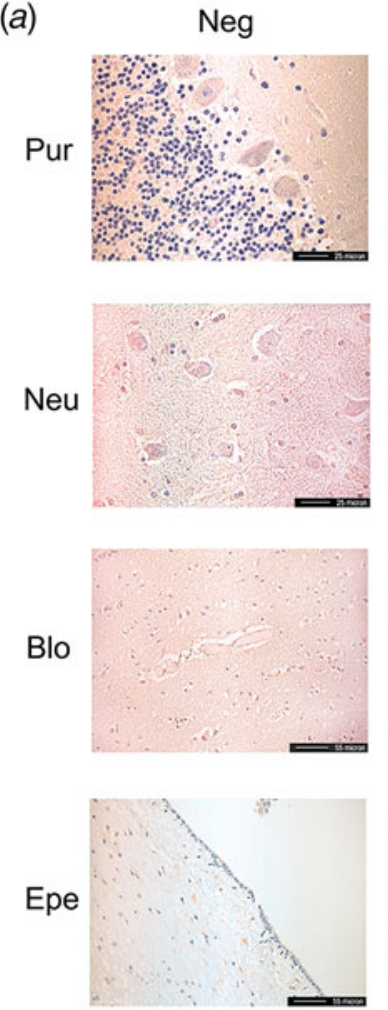

(b)
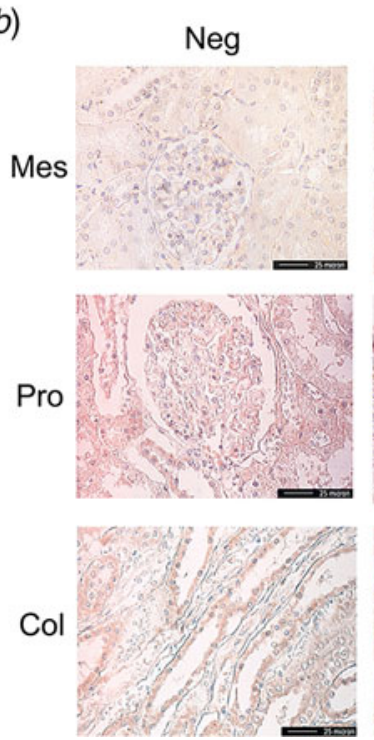

Pos
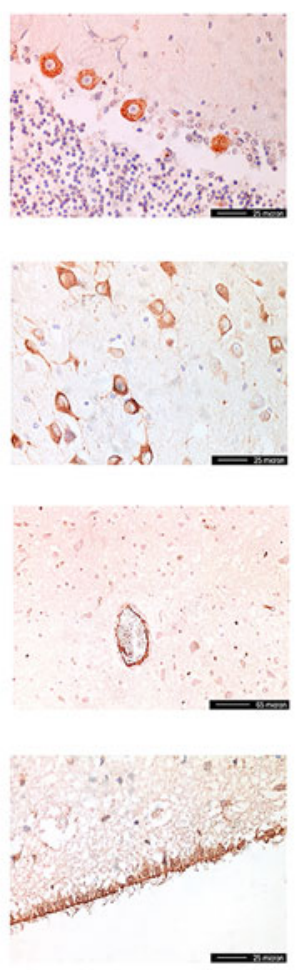

Pos
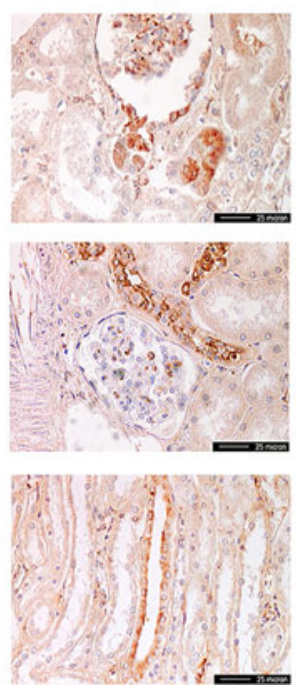
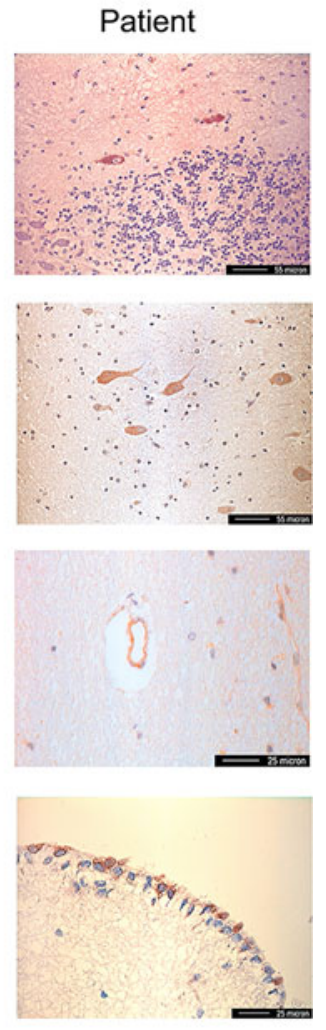

Patient

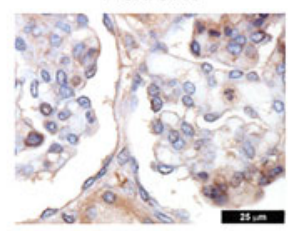

Fig. 1. Localization of Gb3 in (a) human brain and (b) kidney. (a) In some forensic autopsy cases without brain and renal lesions, Gb3 expression was negative (Neg). In other autopsy cases, Gb3 was positive (Pos) in Purkinje cells (Pur) of the cerebellum and neurons (Neu), blood vessels (Blo), and ependymal cells (Epe) around the ventricle. Gb3 was detected in Purkinje cells and neurons, blood vessels, and ependymal cells of a fatal case (Patient). (b) As with the kidney tissues in some forensic autopsy cases, Gb3 was positive (Pos) in mesangial cells (Mes), proximal tubules (Pro) and collecting tubules $(\mathrm{Col})$. Gb3 was negative in other specimens of human kidney (Neg). Gb3 was detected in mesangial cells of a fatal case (Patient).

(Fig. 2). Only in 2008, for patients aged between 5 and 20 years, was HUS significantly associated with female sex (Fig. 2). This study concluded that, in
Japan, adult women had a significantly greater risk of developing HUS. Additionally, in 2006, 2007, 2008 and 2009, the percentage of stool samples 
Table 1. Percentage of autopsy cases indicating positive immunodetection of Gb3

\begin{tabular}{|c|c|c|c|c|c|c|}
\hline & \multicolumn{2}{|c|}{ Infants } & \multicolumn{2}{|c|}{ Adults } & \multicolumn{2}{|l|}{$P$ value } \\
\hline & Male & Female & Male & Female & Adult $v s$. infant & Male $v s$. female \\
\hline \multicolumn{7}{|l|}{ Brain } \\
\hline Purkinje cells & 86 & 100 & 50 & 60 & 0.05 & $0 \cdot 71$ \\
\hline Blood vessels & 43 & 50 & 10 & 10 & $<0.05$ & $1 \cdot 0$ \\
\hline Neurons & 86 & 83 & 10 & 40 & $<0.05$ & $0 \cdot 49$ \\
\hline \multicolumn{7}{|l|}{ Kidney } \\
\hline Mesangial cells & 71 & 100 & 10 & 10 & $<0 \cdot 01$ & 0.73 \\
\hline Proximal tubules & 29 & 17 & 0 & 0 & 0.05 & $1 \cdot 0$ \\
\hline Distal tubules & 14 & 67 & 0 & 40 & $0 \cdot 43$ & $<0.01$ \\
\hline Collecting tubules & 43 & 100 & 20 & 70 & $0 \cdot 28$ & $<0.01$ \\
\hline
\end{tabular}

Table 2. Demographic features, clinical signs, stool culture, and bedridden status for 52 patients infected with STEC O157:H7

\begin{tabular}{|c|c|c|c|}
\hline \multirow[b]{2}{*}{ Factor } & \multicolumn{2}{|c|}{$\begin{array}{l}\text { No. }(\%) \text { of } \\
\text { patients }\end{array}$} & \multirow{2}{*}{$\begin{array}{l}\text { Fisher's exact } \\
\text { test }\end{array}$} \\
\hline & Survived & Died & \\
\hline No. of patients analysed & $45(87)$ & $7(13)$ & \\
\hline \multicolumn{4}{|l|}{ Demographic data } \\
\hline \multicolumn{4}{|l|}{ Age, yr } \\
\hline$\leqslant 85$ & $20(38)$ & $4(7 \cdot 7)$ & $P=0.69$ \\
\hline$>86$ & $25(48)$ & $3(5 \cdot 8)$ & \\
\hline \multicolumn{4}{|l|}{ Sex } \\
\hline Male & $16(31)$ & $0(0)$ & $P=0.09$ \\
\hline Female & $29(56)$ & $7(13)$ & \\
\hline \multicolumn{4}{|l|}{ Bloody diarrhoea } \\
\hline Yes & $16(31)$ & $7(13)$ & $P<0.01$ \\
\hline No & $29(56)$ & $0(0)$ & \\
\hline \multicolumn{4}{|l|}{ Stool culture of STEC } \\
\hline \multicolumn{4}{|l|}{$\mathrm{O} 157: \mathrm{H} 7$} \\
\hline Positive & $32(62)$ & $5(9 \cdot 6)$ & $P=1 \cdot 0$ \\
\hline Negative & $13(25)$ & $2(3 \cdot 8)$ & \\
\hline \multicolumn{4}{|l|}{$\begin{array}{l}\text { Care home for the } \\
\text { elderly }\end{array}$} \\
\hline No. 1 & $35(67)$ & $4(7 \cdot 7)$ & $P=0 \cdot 23$ \\
\hline No. 2 & $10(19)$ & $3(5 \cdot 8)$ & \\
\hline \multicolumn{4}{|l|}{ Bedridden status } \\
\hline $\begin{array}{l}\text { Independent or } \\
\text { partially }\end{array}$ & $38(73)$ & $3(5 \cdot 9)$ & $P<0.05$ \\
\hline Completely & $7(13)$ & $4(7 \cdot 7)$ & \\
\hline
\end{tabular}

which tested positive for the STEC infection were $70 \%, 63 \%, 68 \%$ and $66 \%$ respectively.

\section{DISCUSSION}

This study investigated the localization of the glycosphingolipid receptor Gb3 in human brain and renal tissues removed from 33 autopsy cases in Japan. The occurrence of $\mathrm{Gb} 3$ immunoreactivity in the distal tubules and collecting tubules from female kidneys was greater than in male kidneys. Gb3 immunoreactivity in mesangial cells was more frequent in infant than in adult kidneys. STEC-associated HUS is most prevalent in infants and young children and is the most common cause of acute renal failure in childhood [17]. Chaisri et al. reported that binding of Stx1 and Stx 2 was lower in an adult female patient than a female infant and was limited only to the renal tubules of the adult female, while more binding of Stx 1 and Stx2 (Stxs) was found in renal tubules and glomeruli, including podocytes, mesangial and endothelial cells in the infant female than the adult female [18]. Uchida et al. reported a case of Stxs bound to the distal tubules of an infant female in 1999 [19]. Based on the relationship between mean arterial pressure and renal sympathetic nerve activity, the cause of death in the rabbit model was circulatory failure caused by impairment of the cardiovascular centre in the medulla [20]. Akashi et al. reported that two patients died of intractable tonic-clonic seizures with incomplete HUS in the outbreak at the nursery school in Saitama [7]. Brain dysfunction is an important predictive factor for HUS and mortality in children [21-23]. Consistent with the suggestion that Stxs may have a direct toxic action on the neuron and microvascular endothelia in in the infant brain, the results of this study demonstrates that positive immunodetection of $\mathrm{Gb} 3$ was more frequent in infant brain tissues than in adult brain tissues.

In the outbreak in Kagawa Prefecture in Japan, 425 people had consumed pickled Chinese cabbage at breakfast and dinner. Of these 425 people, 39 patients who developed diarrhoea or bloody diarrhoea, plus 13 
Table 3. Laboratory test results and antibiotic treatment obtained on patients admitted to hospital in care home no. 1 for the elderly

\begin{tabular}{|c|c|c|c|}
\hline & \multicolumn{2}{|c|}{ No. $(\%)$ of patients } & \multirow[b]{2}{*}{ Fisher's exact test } \\
\hline & Survived & Died & \\
\hline No. of patients analysed & $22(85)$ & $4(15)$ & \\
\hline \multicolumn{4}{|l|}{ Test results } \\
\hline \multicolumn{4}{|c|}{ WBC count $>1.02 \times 10^{10}$ cells $/ l^{*}$} \\
\hline Yes & $8(32)$ & $4(16)$ & $P<0.05$ \\
\hline No & $13(52)$ & $0(0)$ & \\
\hline \multicolumn{4}{|c|}{ Platelet count $<1.86 \times 10^{11}$ cells $/ 1^{*}$} \\
\hline Yes & $9(36)$ & $4(16)$ & $P=0 \cdot 10$ \\
\hline No & $12(48)$ & $0(0)$ & \\
\hline \multicolumn{4}{|c|}{ Haemoglobin level $<12 \cdot 1 \mathrm{~g} / \mathrm{dl} *$} \\
\hline Yes & $12(48)$ & $1(4)$ & $P=0.32$ \\
\hline No & $9(36)$ & $3(12)$ & \\
\hline \multicolumn{4}{|c|}{ C-reactive protein $>1.8 \mathrm{mg} / \mathrm{dl}^{\mathrm{a}}$} \\
\hline Yes & $10(40)$ & $2(8)$ & $P=1 \cdot 0$ \\
\hline No & $11(44)$ & $2(8)$ & \\
\hline \multicolumn{4}{|l|}{ Creatinine level $>0.91 \mathrm{mg} / \mathrm{dl}$} \\
\hline Yes & $9(35)$ & $4(15)$ & $P=0 \cdot 10$ \\
\hline No & $13(50)$ & $0(0)$ & \\
\hline \multicolumn{4}{|c|}{ Urea concentration $>19.3 \mathrm{mg} / \mathrm{dl}$} \\
\hline Yes & $9(35)$ & $4(15)$ & $P=0 \cdot 10$ \\
\hline No & $13(50)$ & $0(0)$ & \\
\hline \multicolumn{4}{|c|}{ Admission treatment of patients infected with STEC O157:H7 } \\
\hline \multicolumn{4}{|l|}{ Levofloxacin } \\
\hline Yes & $16(62)$ & $3(12)$ & $P=1 \cdot 0$ \\
\hline No & $6(23)$ & $1(3 \cdot 8)$ & \\
\hline \multicolumn{4}{|l|}{ Fosfomycin } \\
\hline Yes & $16(62)$ & $3(12)$ & $P=1 \cdot 0$ \\
\hline No & $6(23)$ & $1(3 \cdot 8)$ & \\
\hline
\end{tabular}

* Data missing for one patient.

Table 4. Association between sex and renal failure in two outbreaks

\begin{tabular}{llccc}
\hline \hline & \multicolumn{4}{l}{ No. of patients with renal failure/total no. of diarrhoea cases } \\
\cline { 2 - 4 } & $<60 \mathrm{yr}$ & $60-69 \mathrm{yr}$ & $70-79 \mathrm{yr}$ & $\geqslant 80 \mathrm{yr}$ \\
\hline Women & $1 / 19(5 \cdot 3 \%)$ & $2 / 8(25 \%)$ & $2 / 21(9 \cdot 5 \%)$ & $10 / 47(21 \cdot 3 \%)$ \\
Men & $0 / 24(0 \%)$ & $0 / 19(0 \%)$ & $2 / 14(14 \cdot 3 \%)$ & $0 / 10(0 \%)$ \\
\hline \hline
\end{tabular}

asymptomatic patients with STEC in stool specimens were identified. There were 52 patients who were considered to be probable and confirmed cases infected with STEC. In outbreaks of STEC, severe bloody diarrhoea in elderly patients is fatal even without detecting STEC in stool specimens. Ikeda et al. reported that STEC inpatients who took fosfomycin within the first 2 days of illness developed HUS significantly less often than those who did not [24]. In care home no. 1 in the Kagawa outbreak, fosfomycin and levofloxacin also had no effect on patients upon admission to hospital.

In laboratory tests, WBC count $>10^{10}$ cells/l was significantly associated with death caused by STEC. Ikeda et al. reported that clinical features associated with hospitalized patients who developed HUS compared to those without HUS included C-reactive protein $(\mathrm{CRP})$ level $>1.2 \mathrm{mg} / \mathrm{dl}$, WBC count $>1.1 \times 10^{10}$ cells $/ 1$, and temperature $>38.0{ }^{\circ} \mathrm{C}$ [25]. The results of 


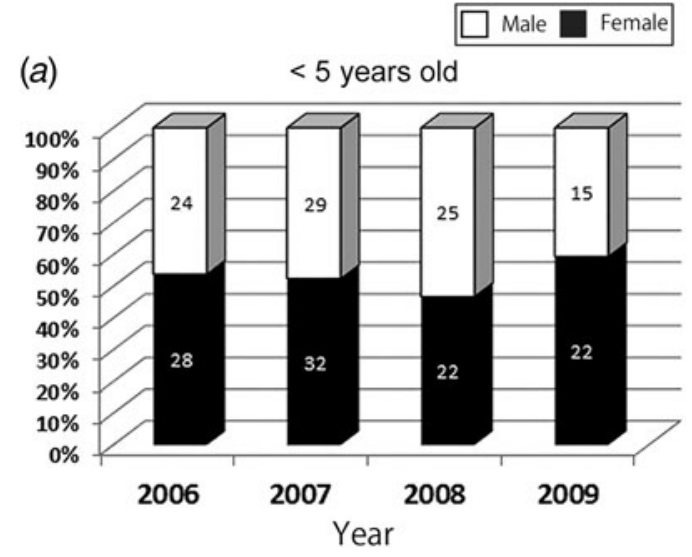

(b)

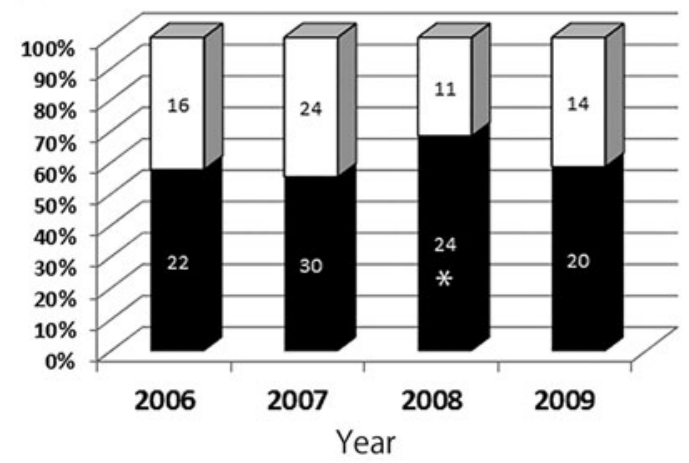

$(c)$

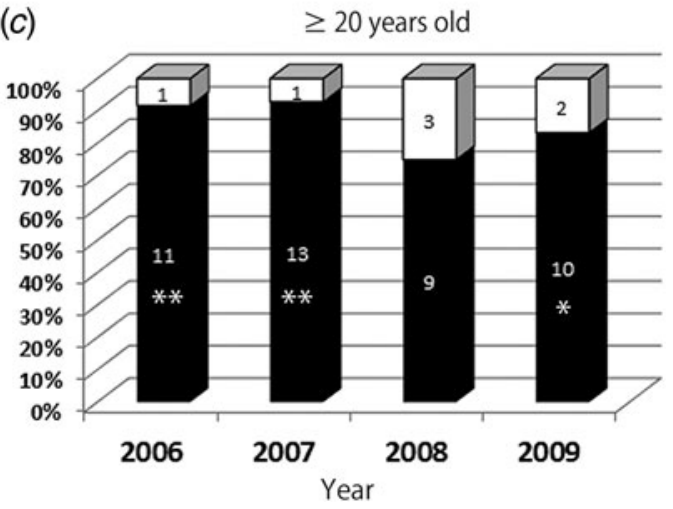

Fig. 2. Sex distribution of haemolytic uraemic syndrome (HUS) patients infected with STEC in Japan. In patients aged $\geq 20$ years, females were significantly more at risk of developing HUS in 2006, 2007 and 2009 (** $P<0.01$, $* P<0 \cdot 05$ ). In patients aged 5 to $<20$ years, females were at significantly greater risk of developing HUS in 2008 (* $P<0 \cdot 05)$.

the present study revealed that like those reported for HUS, WBC count may be an important indicator for progression to death; however, CRP was not significantly associated with death caused by STEC in Kagawa Prefecture.

Su \& Brandt reported case-fatality rates for STEC infection of $3-36 \%$ in elderly residents of nursing homes
[26]. In the United States from 1982 to 2002, gastrointestinal infection progressed to HUS in $4 \%$ of patients and death in $0.5 \%$ [27]. In our study, the mortality rate in symptomatic patients was $18 \%(7 / 39)$. However, in residents aged $>65$ years, there was no association between age and death caused by STEC infection.

To confirm the risk factors for STEC infection, a retrospective analysis of predictors of renal failure in infected patients admitted to hospital was performed during the Kagawa and Tochigi Prefecture outbreaks in Japan. The analysis revealed that female sex was a risk factor for renal failure including HUS associated with STEC infection (Table 4). Rowe et al. reported that girls had a significantly greater risk than boys of developing haemolytic anaemia after STEC O157: $\mathrm{H} 7$ infection in a retrospective cohort study of 72 children [28]. However, previous reports on outbreaks of STEC have not established a link between HUS including renal failure and the sex of the patient. In the Sakai City STEC outbreak, $98 \%$ of HUS cases occurred in patients aged $<12$ years [10]. In the Central Scotland outbreak, $22 \%$ of cases involved HUS in patients aged $<15$ years [11]. In this study, renal failure, caused by STEC, occurred in patients aged $\geqslant 58$ years. In older populations, adult females may have a greater risk factor of contracting HUS. Forensic autopsies highlighted that Gb3 immunoreactivity in distal tubules and collecting tubules was more frequent in females than in males. This evidence may be associated with the risk of HUS and renal failure being more frequent in females. Generally, infants were more likely to be susceptible to HUS caused by STEC than adults, and adult HUS cases were rare. Therefore, as our next step, gender factor in adult HUS cases in Japan were analysed.

In this study, data from surveillance conducted by the IDSC between 2006 and 2009, revealed that detection rates of STEC infections in stool samples in Japanese HUS cases were high, measuring between $63 \%$ and $70 \%$. Their data show that female sex is associated more with the risk of HUS than age in the adult population. The present study concludes that female sex is a risk factor for HUS caused by STEC infection in Japan.

In 2008 and 2009, HUS cases linked to beef were $39 \%$ and $33 \%$, respectively. However, there is no evidence to suggest that adult females ate more beef than males during this period.

Recently, it has been suggested that there is an association between HUS and gender. In 2014, an outbreak of STEC O157:H7 infection occurred at a 
fireworks display in Sizuoka Prefecture, Japan. Lightly pickled cucumbers consumed from mobile kitchens were identified as the source of infection after an epidemiological study was performed. Five hundred and thirteen patients developed diarrhoea or bloody diarrhoea, of which five developed HUS. All HUS cases were female (aged 1, 5, 6, 9, 19 years). The outbreak strongly supports the hypothesis, that in Japan, females are at greater risk of developing HUS than males.

The research does not take into account the effect that the food vehicle or eating habits of the sexes played in the transmission of Stx, as no accurate data were available. However, it is felt that the importance of the food vehicle in this study was mitigated as food servings and portions were similar in all the HUS cases due to the outbreak occuring in three care homes where food servings were similar. The adverse effects should be further investigated in future studies.

\section{ACKNOWLEDGEMENTS}

This work was supported by grants-in-aid for Scientific Research as a part of the US-Japan Cooperative Medical Science Program (851114300001) from the Ministry of Health, Labour and Welfare of Japan

\section{DECLARATION OF INTEREST}

None.

\section{REFERENCES}

1. Karch H, et al. The enemy within us: lessons from the 2011 European Escherichia coli O104:H4 outbreak. EMBO Molecular Medicine 2012; 4: 841-848.

2. Rasko DA, et al. Origins of the E. coli strain causing an outbreak of hemolytic-uremic syndrome in Germany. New England Journal of Medicine 2011; 365: 709-717.

3. Hebbelstrup Jensen B, et al. Epidemiology and clinical manifestations of enteroaggregative Escherichia coli. Clinical Microbiology Reviews 2014; 27: 614-630.

4. Frank C, et al. Epidemic profile of Shiga-toxin-producing Escherichia coli O104:H4 outbreak in Germany. New England Journal of Medicine 2011; 365: 1771-1780.

5. Werber D, et al. Associations of age and sex with the clinical outcome and incubation period of Shiga toxinproducing Escherichia coli O104:H4 infections, 2011. American Journal of Epidemiology 2013; 178: 984-992.

6. Gasser C, et al. Hemolytic-uremic syndrome: bilateral necrosis of the renal cortex in acute acquired hemolytic anemia [in German]. Schweizerische Medizinische Wochenschrift 1955; 85: 905-909.
7. Akashi S, et al. A severe outbreak of haemorrhagic colitis and haemolytic uraemic syndrome associated with Escherichia coli O157:H7 in Japan. European Journal of Pediatrics 1994; 153: 650-655.

8. Michino H, et al. Massive outbreak of Escherichia coli O157:H7 infection in schoolchildren in Sakai City, Japan, associated with consumption of white radish sprouts [see comments]. American Journal of Epidemiology 11999; 150: 787-796.

9. Fukushima H, et al. Clinical experiences in Sakai City Hospital during the massive outbreak of enterohemorrhagic Escherichia coli O157 infections in Sakai City, 1996. Pediatrics International 1999; 41: 213-217.

10. Okajima S. An outline of 1996 outbreak related with STEC O157 infection in Sakai City. Sakai City committee report of STEC O157 outbreak in Japan, 1997.

11. Dundas S, et al. The central Scotland Escherichia coli O157:H7 outbreak: risk factors for the hemolytic uremic syndrome and death among hospitalized patients. Clinical Infectious Diseases 2001; 33: 923-931.

12. Endo Y, et al. Site of action of a Vero toxin (VT2) from Escherichia coli O157:H7 and of Shiga toxin on eukaryotic ribosomes. RNA N-glycosidase activity of the toxins. European Journal of Biochemistry 1988; 171: 45-50.

13. Lingwood CA, et al. Glycolipid binding of purified and recombinant Escherichia coli produced verotoxin in vitro. Journal of Biological Chemistry 1987; 262: 8834-8839.

14. Mangeney M, et al. Apoptosis induced in Burkitt's lymphoma cells via Gb3/CD77, a glycolipid antigen. Cancer Research 1993; 53: 5314-5319.

15. Ohyama C, et al. Immunohistochemical study of globotriaosyl ceramide $(\mathrm{Gb} 3)$ in testicular tumors [in Japanese]. Nippon Hinyokika Gakkai Zasshi 1993; 84: 1308-1315.

16. Yokobayashi K, et al. Prospective cohort study of fever incidence and risk in elderly persons living at home. BMJ Open 2014; 4: e004998.

17. Scheiring J, Andreoli SP, Zimmerhackl LB. Treatment and outcome of Shiga-toxin-associated hemolytic uremic syndrome (HUS). Pediatric Nephrology 2008; 23: 1749-1760.

18. Chaisri U, et al. Localization of Shiga toxins of enterohaemorrhagic Escherichia coli in kidneys of paediatric and geriatric patients with fatal haemolytic uraemic syndrome. Microbial Pathogenesis 2001; 31: 59-67.

19. Uchida H, et al. The detection of Shiga toxins in the kidney of a patient with hemolytic uremic syndrome. Pediatric research 1999; 45: 133-137.

20. Yamada Y, et al. Brainstem mechanisms of autonomic dysfunction in encephalopathy-associated Shiga toxin 2 intoxication. Annals of Neurology 1999; 45: 716-723.

21. Rooney JC, Anderson RM, Hopkins IJ. Clinical and pathological aspects of central nervous system involvement in the haemolytic uraemic syndrome. Australian Paediatrc Journal 1971; 7: 28-33.

22. Siegler RL. The hemolytic uremic syndrome. Pediatric Clinics of North America 1995; 42: 1505-1529.

23. Upadhyaya K, et al. The importance of nonrenal involvement in hemolytic-uremic syndrome. Pediatrics 1980; 65: 115-120. 
24. Ikeda K, et al. Effect of early fosfomycin treatment on prevention of hemolytic uremic syndrome accompanying Escherichia coli O157:H7 infection. Clinical Nephrology 1999; 52: 357-362.

25. Ikeda K, et al. Predictors for the development of haemolytic uraemic syndrome with Escherichia coli O157:H7 infections: with focus on the day of illness. Epidemiology and Infection 2000; 124: 343-349.
26. Su C, Brandt LJ. Escherichia coli O157:H7 infection in humans. Annals of Internal Medicine 1995; 123: 698-714.

27. Rangel JM, et al. Epidemiology of Escherichia coli O157:H7 outbreaks, United States, 1982-2002. Emerging Infectious Diseases 2005; 11: 603-609.

28. Rowe PC, et al. Haemolytic anaemia after childhood Escherichia coli $\mathrm{O} 157: \mathrm{H} 7$ infection: are females at increased risk? Epidemiology and Infection 1991; 106: 523-530. 